\title{
16. Electric Responses of Auditory Neurons in Cat to Sound Stimulation
}

\author{
By Tadaaki SumI \\ Department of Physiology, Faculty of Medicine, Tottori University \\ Yasuji KATSUKI and Heiichi UCHIYAMA \\ Department of Physiology, Tokyo Medical and Dental University \\ (Comm. by T. Furuhata, M.J.A., Jan. 12, 1956)
}

Tasaki' $\mathrm{s}^{1)}$ application of the superfine microelectrode method to the recording of auditory nerve impulse in guinea pig was successful in obtaining some important results, but was confined in its peripheral neurons. ${ }^{2)}$ The present report deals with the results obtained in cat by the same method in more extensive regions of auditory neurons: the cochlear nerve bundle, the dorsal cochlear nucleus, the trapezoid body, and the inferior colliculus.

\section{Method}

More than 100 cats were anesthetized with dial or nembutal, or decerebrated. To keep the local blood circulation of brain as intact as possible, the tissues obstructing the introduction of an electrode were carefully thrusted aside by a spatula. The electrodes used were $3 \mathrm{M}-\mathrm{KCl}$ capillary microelectrodes with ohmic resistances between 30 and $50 \mathrm{M} \Omega$, and the electronmicroscopic examination of such an electrode revealed its tip-diameter to be less than $0.2 \mu$. As main amplifiers an $\mathrm{RC}$ and, if necessary, a high gain $\mathrm{DC}$ amplifier were employed simultaneously. The sound sources were two loud-speakers in combination, whose response curve was almost flat within $\pm 10 \mathrm{db}$ from 300 to $20000 \mathrm{cps}$. All the experiments were performed in a soundproof room where the noise level was $0 \mathrm{db}$, the temperature being kept constant by air conditioning at about $28^{\circ} \mathrm{C}$.

\section{Results}

Most of spike discharges obtained were monophasic and positive, measuring from 1 to $5 \mathrm{mV}$. The recording from the same element could be made usually for scores of minutes and under the most favorable condition for one hour.

Spontaneous discharges

All the elements dealt with in the present report showed spontaneous discharges, some of which were of a very low rate and some others of a very high rate over 100 per second. These discharges were easily distinguished from those of other tracts by their charac- 
teristic irregularity. The elements that responded to high frequency sounds showed very high rate discharges, and discharges recorded from the peripheral region were often correlated with respiratory movement.

\section{Response areas to continuous tone}

The response areas of individual elements were obtained in the four regions. Fig. 1 shows those from the cochlear nerve bundle. The latencies of these elements to click stimulation ranged from less than 1 to 2 msec. In view of the result, it can not be denied that the secondary neurons are also comprised in them. As shown in the figure the response areas are different from those obtained by Galambos and Davis, ${ }^{3)}$ and similar to Tasaki's results in guinea pig. ${ }^{12}$ In each element the area shows a sharp cut-off at the side of high frequency and a gradual rise in threshold toward the low frequency

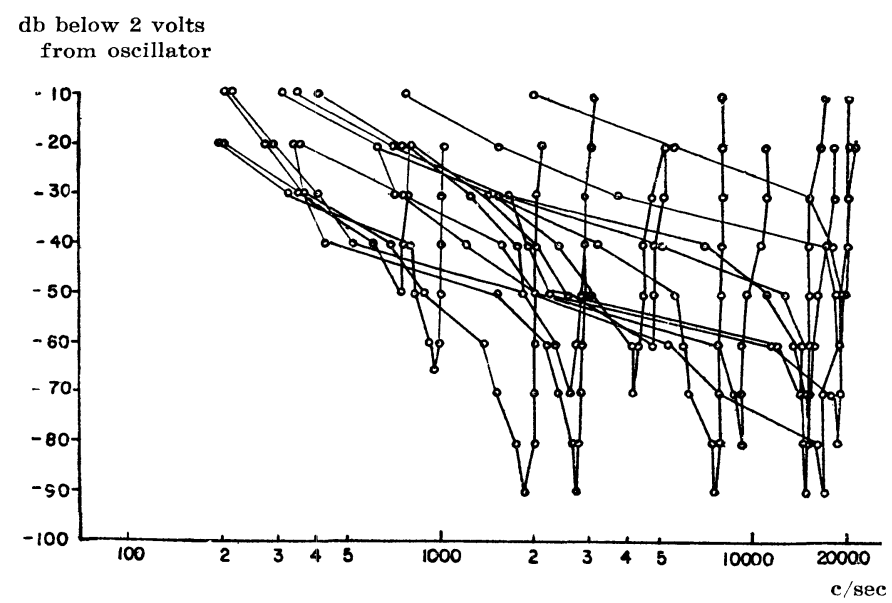

Fig. 1. Response areas from cochlear nerve bundle

side. In other words all the elements can respond to tones of sufficient intensity in very wide ranges below the cut-off frequency. In elements of the same characteristic frequency the thresholds were not consistent with each other. The most sensitive threshold obtained at the neighborhood of $2000 \mathrm{cps}$ coincides with that of human, although this animal is much more sensitive to high tones over $10000 \mathrm{cps}$. The spike frequency was linearly correlated with the sound intensity in $\mathrm{db}$ in a certain range.

Fig. 2 illustrates response areas at the level of dorsal cochlear nucleus. ${ }^{3)}$ There can be seen two different groups. One is the same as in Fig. 1, whereas the other has narrower frequency ranges. When the animals were under narcotics wider ranges were shown in most elements, while in decerebration narrower ranges were obtained as well. The latencies to click measured from less than 1 to $5 \mathrm{msec}$ : 
From this result it is very conceivable that the two groups described above may belong to the primary and the secondary neurons respectively. This is supported by the fact that some of these elements with longer latencies showed inhibitory phenomena to other sounds

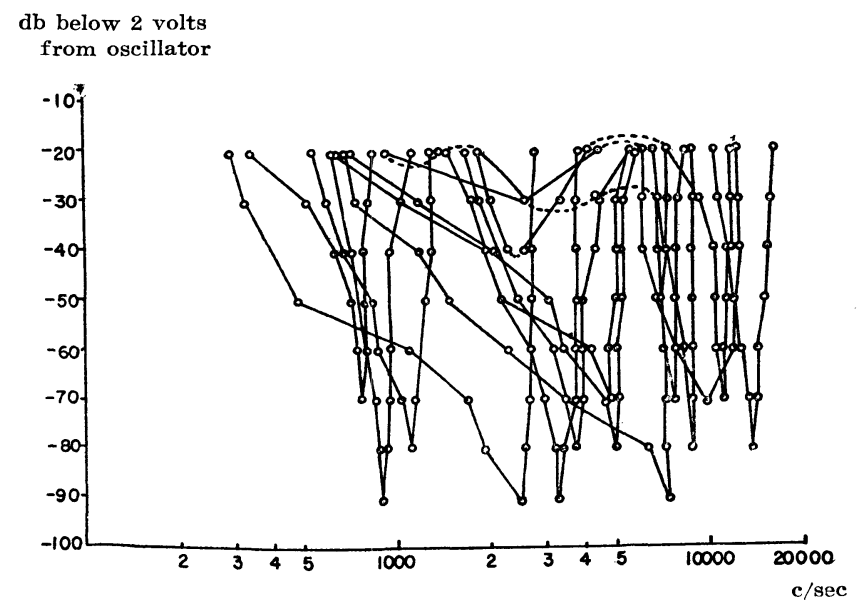

Fig. 2. Response areas from the region of dorsal cochlear nucleus The parts shown with dotted line are not actually measured.

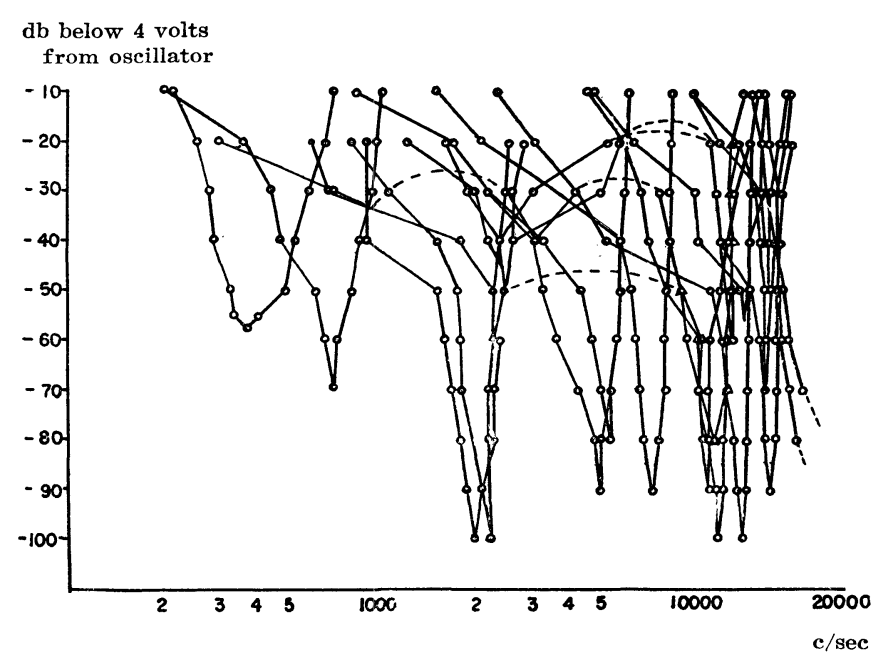

Fig. 3. Response areas from trapezoid body

delivered simultaneously, as was observed by Galambos. ${ }^{4)}$ Details thereof will be described elsewhere.

The response areas of Fig. 3 are those obtained at the level of trapezoid body..$^{5)}$ Most elements are provided with narrow areas, similar to the second type at the level of dorsal cochlear nucleus Some elements that responded to very high frequency tones have tonal gaps, and at the level of dorsal cochlear nucleus these gaps 
were still found in a few cases. Latencies to click ranged from 2 to 5 msec.

In Fig. 4 are shown response areas at the level of the inferior colliculus. ${ }^{6}$ ) Most of them were recorded in the region between the inferior colliculus and the medial geniculate body at the side opposite to the ear exposed directly to the sound sources. Comparing with that at lower levels, recording from this region was pretty difficult. As seen in the figure all the elements have very narrow ranges and moreover many of them have high thresholds. The high threshold elements responded continuously during the stimulation but with

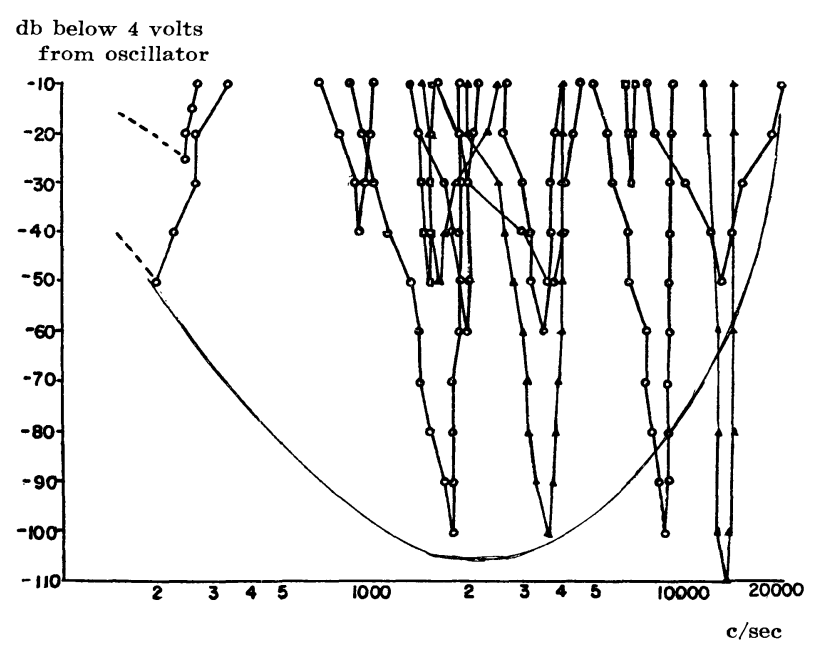

Fig. 4. Response areas from inferior collicular region The curve without circle shows human threshold.

rapid adaptation, and the increase of discharge frequency with the increased stimulus intensity was not so large as that at the lower level. In contrast, elements with low thresholds discharged only at the onset of the change of sound frequency. These were also responsive to click or tone pips, with the latencies varying between 5 and $8 \mathrm{msec}$. However the number of spike in response to such brief stimulation was three at most. This is different from that at the lower level, where the elements responded to the same stimulation with many spikes. Since the result at this level was found to be remarkably influenced by the depth of anesthesia, the response pattern was carefully examined by some different methods. In order to verify the reliability of those results the average human threshold of audibility obtained under the same condition has been drawn together in the figure.

\section{Masking phenomenon}

Tasaki and Davis reported that, in guinea pig, discharges by 
brief sound of both the primary ${ }^{1)}$ and the secondary cochlear neurons $^{2)}$ were never inhibited by the second sound. In cats, however, a train of spike discharges by click was inhibited by pure tones, noise, and also human voice. At any level, even at the most peripheral region, the number of spike in a train decreased as the second stimulus became intense, and at a certain intensity the response to click came to be completely masked by the appearance of responses to the second stimulation. The above difference between Tasaki's and this results might be attributed to the difference of animal species.

Tonotopic localisation

Clear tonotopic localisation is known at both extreme ends of the auditory system: the cochlea and the cortex. However in regions between them it has still not been identified. During the process of the present experiment it was very often encountered that, when records were obtained from several elements in a very restricted area, their response patterns were very similar to each other. In one case at the level of trapezoid body, it was found that several elements which rosponded to very high tones over 20000 cps were situated rostrally while those to very low tones below $300 \mathrm{cps}$ were located caudally and between the two groups elements to $5000 \mathrm{cps}$ and $3000 \mathrm{cps}$ stood side by side. These findings may strongly suggest the tonotopic localisation being en route.

Responses at the higher level i.e. the geniculate body and the cortex are now under investigation.

This work was aided by a grant from the Ministry of Education of Japan.

\section{References}

1) Tasaki, I.,: J. Neurophysiol., 17, 97-122 (1954).

2) Tasaki, I., and Davis, H.,: J. Neurophysiol., 18, 151-158 (1955).

3) Galambos, R., and Davis, H.,: J. Neurophysiol., 6, 39-57 (1943).

4) Galambos, R., and Davis, H.,: J. Neurophysiol., 7, 287-303 (1944).

5) Hilali, S., and Whitfield, I. C.,: J. Physiol., 122, 158-171 (1953).

6) Thurlow, W. R., Gross, N. B., Kemp, E. H., and Lowy, K.,: J. Neurophysiol., 14 289-304 (1951). 